\title{
COMPORTAMENTO DE MUDAS DE Bambusa vulgaris Schrad. EX J.C. Wendl SUBMETIDAS AO ESTRESSE HÍDRICO E SALINO, UTILIZANDO ÁGUA RESIDUÁRIA DA PISCICULTURA
}

\author{
Cleyton dos Santos Souza ${ }^{*}$, Valéria Nayara Silva oliveira ${ }^{2}$, Elaine Cristina Alves da Silva ${ }^{3}$, Lidiane Martins \\ Moura Ferreira ${ }^{4}$, Maria Janaina Nascimento Silva ${ }^{2}$, Poliana coqueiro dias Araújo ${ }^{5}$
}

\author{
${ }^{1}$ Graduando em Engenharia Florestal, Universidade Federal Rural do Semi Árido (UFERSA), Av. Francisco Mota, 572 - Bairro Costa e \\ Silva, Mossoró/RN - CEP: 59.625-900 \\ 2Mestrandos em ambiente, tecnologia e sociedade, Universidade Federal Rural do Semi Árido (UFERSA), Av. Francisco Mota, 572 - \\ Bairro Costa e Silva, Mossoró/RN - CEP: $59.625-900$ \\ ${ }^{3}$ Doutora em Biotecnologia, Universidade Federal Rural de Pernambuco (UFRPE), Rua Dom Manuel de Medeiros, s/n, Dois Irmãos - \\ Recife/PE - CEP: 52.171-900 \\ ${ }^{4}$ Mestre em química analítica, Universidade Federal do Amazonas, Av. General Rodrigo Octavio Jordão Ramos 1200 - coroado I, \\ Manaus - AM, 69067-005 \\ ${ }^{5}$ Professora titular, Departamento de Ciências Agronômicas e Florestais, Universidade Federal Rural do Semi Árido (UFERSA), Av. \\ Francisco Mota, 572 - Bairro Costa e Silva, Mossoró/RN - CEP: 59.625-900
}

*Autor para correspondência: Cleyton dos Santos Souza, klaytonsantossouzaprivado@gmail.com

RESUMO: Bambusa vulgaris schrad. Exj.c. wendl é uma espécie de bambu cultivada em diversas regiões do Brasil. Contudo, há regiões com déficit hídrico pronunciado e o reuso de água é uma importante alternativa com vistas à produção agrícola e florestal. O reuso de efluentes de viveiros de peixes para a irrigação é uma prática agrícola possivel desde que haja tolerância da planta a salinidade. Objetivou-se neste trabalho, verificar a tolerância de mudas de $B$. vulgaris ao estresse hídrico e salino utilizando água residuária da piscicultura na irrigação. Realizado na UFERSA - Mossoró, sob o delineamento experimental em DIC disposto em esquema fatorial 3x5. Foi utilizada água residuária com porcentagem de 0,10,15, 20 e 25\% de salinidade. Foram analisadas as seguintes variáveis: fitomassa seca da parte aérea e do sistema radicular, concentração de clorofila $a$, clorofila $b$ e carotenoides, determinação dos teores de macronutrientes, análises de solo e água de irrigação. A variável fitomassa apresentou decréscimo em relação aos demais parâmetros analisados e as mudas de $B$. vulgaris mostraram mais sensíveis ao estresse hídrico do que à salinidade. Conclui-se que, a água residuária da piscicultura apresenta-se como alternativa para irrigação, entretanto, mudas de Bambusa vulgaris vittata não resistem a falta de irrigação por períodos superiores a 14 dias.

PALAVRAS CHAVE: Tolerância; Salinidade; Semiárido; Bambu.

\section{BEHAVIOR OF CHANGES OF Bambusa vulgaris SCHRAD. EX J.C. WENDL SUBMITTED TO WATER AND SALINE STRESS, USING WASTE RESIDUE WATER FROM PISCICULTURE}

ABSTRACT: Bambusa vulgaris schrad. Ex.j.c. Wendl is a species of bamboo grown in several regions of Brazil. However, there are regions with pronounced water deficit and water reuse is an important alternative for agricultural and forestry production. Reuse of fish pond effluents for irrigation is a feasible agricultural practice as long as there is plant tolerance to salinity. The objective of this work was to verify the tolerance of $B$. vulgaris seedlings to water and saline stress using pisciculture residual water in irrigation. Performed at UFERSA - Mossoró, under the experimental design in DIC arranged in a $3 \times 5$ factorial scheme. Residual water was used with percentage of $0,10,15,20$ and $25 \%$ of salinity. The following variables were analyzed: dry matter of the aerial part and the root system, chlorophyll a concentration, chlorophyll b and carotenoids, determination of macronutrient contents, soil and irrigation water analysis. The phytomass variable presented a decrease in relation to the others analyzed and the seedlings of $B$. vulgaris were more sensitive to water stress than to salinity. It is concluded that, the pisuculture wastewater presents as an alternative for irrigation, however, seedlings of Bambusa vulgaris vittata do not resist the lack of irrigation for periods superior to 14 days.

KEY WORDS: Tolerance; Salinity; Semi-arid; Bamboo. 


\section{INTRODUÇÃO}

O semiárido Brasileiro está entre as regiões secas mais populosas do mundo, onde envolve quase todos os estados do Nordeste (Piauí, Ceará, Rio Grande do Norte, Paraíba, Pernambuco, Alagoas, Sergipe e Bahia), exceto o Maranhão, e inclui o norte de Minas Gerais (Vieira, 2009). Em regiões semiáridas, o déficit hídrico é característico e a população que vive e produz nesses locais procura formas alternativas para subsistência. 0 desenvolvimento dessa região é proveniente, principalmente, da produção agrícola, a qual contribui para a geração de emprego e 0 aumento da renda.

Para suprir a indisponibilidade hídrica com vistas a irrigação nesta região, é utilizada água de poços as quais, na maioria das vezes, apresentam elevadas concentrações de sais, sendo assim considerada de baixa qualidade (Silva, 2014). 0 uso destas águas salobras para a criação de peixe de água doce vem se tornando uma opção viável para a população como uma fonte de renda. Em contrapartida, devido aos dejetos e aos restos de ração da alimentação dos peixes, é necessária à sua troca, o que resulta em um rejeito chamado de água residuária (Guimarães et al., 2016).

0 reuso de água proveniente da piscicultura na irrigação é uma alternativa interessante, pois além de benefícios ambientais, uma vez que seu descarte no solo é evitado, também apresenta benefícios econômicos, pois não é necessário buscar outras fontes hídricas. Entretanto, deve-se ter atenção na produção agrícola, uma vez que, a depender da origem destas águas, estas podem apresentar contaminantes, sendo tóxicos para as plantas. A integração do uso de água residuária da piscicultura com a agricultura vem apresentando êxito no cultivo de diversas espécies agrícolas, tais como alface (Silva et al., 2014), coentro e cebolinha (Baioni et al., 2017), bem como em essências florestais (Almeida et al., 2017).

Com vistas a ampliar o cultivo de outras espécies florestais, em 2011, foi instituída a Política Nacional de Incentivo ao Manejo Sustentado e ao Cultivo do Bambu, que tem como objetivo o desenvolvimento da cultura do bambu no Brasil por meio de ações governamentais e de empreendimentos privados, e dentre as diretrizes, tem a valorização do bambu como agro-silvo-cultural (Brasil, 2011).

Os bambus são gramíneas perenes, pertencente à família Poacea, subfamília
Bambusoideae. Existem 1641 espécies distribuídas em 120 gêneros, as quais são encontradas em todos os continentes, exceto na Europa e Antártida (Pereira; Beraldo, 2008). No Brasil, são encontrados em todas as regiões, possuem 35 gêneros distribuídos em 258 espécies. A espécie mais plantada no Brasil é a $B$. vulgaris, conhecida também como bambu amarelo ou bambu tigre, a qual possui diversas aplicações na culinária, no paisagismo, no artesanato, na produção de carvão, instrumentos musicais e móveis (Figueiras e Viana, 2017). Seu maior uso se dá na produção de papel para embalagem, devido a sua constituição de $40 \%$ de fibras longas alinhadas, que são ricas em lignina resistente (Santana et al., 2017). A diversidade de uso do bambu e a sua ampla distribuição geográfica possibilita 0 acesso a genótipos com adaptabilidade e estabilidade a diferentes condições de produção (Almeida e Silva Júnior, 2017), o que pode viabilizar a introdução do bambu em regiões semiáridas com vistas a produção por pequenos agricultores e empresas.

Outro fator importante que deve ser considerado na introdução do bambu em regiões semiáridas é a falta regular de chuvas e, especialmente no Brasil, a salinidade da água e dos solos, ocasionada principalmente pelo material de origem das rochas da região. De acordo com Silva etal. (2014), a demanda por água para irrigação em regiões semiáridas tem levado a utilização da maioria das fontes hídricas disponíveis na região, o que reflete na utilização de águas com diferentes níveis de salinidade pelos produtores rurais.

O uso de água com excesso de sais pode limitar a produção agrícola em decorrência do efeito osmótico e a ocorrência de toxicidade iônica e desequilíbrios nutricionais (Assis Júnior et al., 2007). 0 excesso de sais ocasionado pelo estresse salino pode alterar as funções fisiológicas e bioquímicas das plantas, resultando em distúrbios nas relações hídricas e alterações na absorção e na utilização de nutrientes essenciais. Uma das alternativas para o uso dessas águas de elevada salinidade é a sua mistura com água de baixa concentração de sais, sendo este um manejo estratégico para incentivar a inserção dessas águas na produção vegetal em regiões que sofrem com estiagens prolongadas (Silva et al., 2014).

Para verificar a tolerância do $B$. vulgaris em regiões com escassez de água, é necessário submetêlo a condição que pode ser encontrada no campo. Sendo assim, o objetivo deste trabalho foi avaliar o 
comportamento de mudas de $B$. vulgaris ao estresse hídrico e salino utilizando água residuária proveniente de tanques de piscicultura.

\section{MATERIAL E MÉTODOS}

O experimento foi conduzido no Departamento de Ciências Agronômicas e Florestais da Universidade Federal Rural do Semi Árido (UFERSA), em Mossoról $\mathrm{RN}$, em setembro de 2018. O município de Mossoró situa-se a $5^{\circ} 11^{\prime}$ de latitude Sul e $37^{\circ} 20^{\prime}$ de longitude Oeste e altitude de $18 \mathrm{~m}$. Segundo Santos et al. (2014), o clima local é DdAa', ou seja, semiárido, megatérmico e com pequeno ou nenhum excesso de água durante 0 ano e, seco, muito quente, com duas estações climáticas: uma seca, que geralmente compreende 0 período de junho a janeiro e uma chuvosa, entre os meses de fevereiro a maio.

As mudas de $B$. vulgaris utilizadas foram obtidas por propagação vegetativa, produzidas a partir de touceiras com idade média de 12 anos, sadias e vigorosas. 0 método de produção das mudas seguiu as recomendações propostas por Pereira e Beraldo (2008). Para a produção das mudas foram utilizados recipientes de $3 \mathrm{~L}$ de capacidade, contendo como substrato terra de subsolo, foram retiradas amostras do substrato e da água residuária, e encaminhadas para o Laboratório de Análise de Solo Água e Planta (LASAP) da Universidade Federal rural do Semiárido (UFERSA) para fazer a análise química e física. $O$ resultado desta análise inicial encontra-se na Tabela 1.

Tabela 1. Análise da fertilidade do substrato utilizado na produção das mudas pré-formadas de $B$. vulgaris.

\begin{tabular}{|c|c|c|c|c|c|c|c|c|c|c|c|c|c|}
\hline \multirow{2}{*}{ Ident } & \multirow{2}{*}{$\begin{array}{c}N \\
(g / k g)\end{array}$} & \multirow{2}{*}{$\begin{array}{c}\mathrm{pH} \\
\text { (Água) }\end{array}$} & \multirow{2}{*}{$\begin{array}{c}\mathrm{CE} \\
(\mathrm{ds} / \mathrm{m})\end{array}$} & \multirow{2}{*}{$\begin{array}{c}\text { Mat.Org } \\
(\mathrm{g} / \mathrm{kg})\end{array}$} & $P$ & $\mathrm{~K}^{+}$ & $\mathrm{Na}^{+}$ & $\mathrm{Ca}^{2+}$ & SB & $\mathrm{T}$ & CTC & V & PST \\
\hline & & & & & \multicolumn{3}{|c|}{$\left(\mathrm{mg} / \mathrm{dm}^{3}\right)$} & \multicolumn{4}{|c|}{$\left(\mathrm{cmolc} / \mathrm{dm}^{3}\right)$} & \multicolumn{2}{|c|}{$(\%)$} \\
\hline Solo 1 & 1,75 & 7,70 & 1,93 & 6,90 & 1610,3 & 603,9 & 4,50 & 1,10 & 12,35 & 12,35 & 12,35 & 100 & 21 \\
\hline
\end{tabular}

${ }^{*} \mathrm{Mg}$ e alumínio foram removidos da tabela pois os valores obtidos na análise foram zero.

Ident=identificação; $\mathrm{N}=$ nitrogênio total; ph= Potencial Hidrogeniônico; $\mathrm{CE}=$ condutividade elétrica; $\mathrm{P}=$ fósforo; $\mathrm{K}+=$ íon potássio; Na+ = íon sódio; $\mathrm{Ca2}+=$ ín cálcio; $\mathrm{SB}=$ soma de Bases; $\mathrm{T}=\mathrm{Ctc}$ potencial a ph 7,0; CTC= Capacidade de Troca de cátions; V= Percentagem de saturação por bases da CTC a pH 7,0; PST= Porcentagem de Sódio Trocável.

As análises foram realizadas no LASAP utilizando a metodologia sugerida pela Embrapa (2006), que são feitas por via úmida ácida, e o fósforo extraído por uma solução de ácido forte usando Melich 1.

0 delineamento experimental utilizado foi de blocos casualizados, em esquema fatorial $3 \times 5$, correspondendo três regimes hídricos (Rega a cada 2 dias; rega a cada 7 dias e rega a cada 14 dias) e cinco concentrações de água residuária na irrigação $(0,10$, 15,20 e $25 \%$ ) e seis repetições, aplicando o volume de $500 \mathrm{ml}$ de água em todos os tratamentos em cada irrigação. Aágua residuária foi proveniente de tanques de piscicultura com 20 ppm de salinidade, coletada no setor de Pesca da UFERSA a qual foi diluída em água de abastecimento fornecida pela universidade. 0 experimento teve duração de 28 dias, após o início das irrigações com as respectivas concentrações da água residuária, provenientes de tanques experimentais de Tilápia, utilizando ração convencional.

As avaliações das concentrações de clorofila a, clorofila b e carotenóides seguiram os padrões sugeridos por (Lischentaler e Buschmam, 2001). Para a determinação dos teores de macronutrientes, as mudas foram separadas em folhas e raízes, colocadas para secar em estufa de secagem com ventilação forçada por $72 \mathrm{~h}$ a $65^{\circ} \mathrm{C}$. Após o processo de secagem, as amostras foram encaminhadas ao Laboratório de Engenharia Florestal da UFERSA, onde foram trituradas em moinho de facas tipo Willey e acondicionadas em frascos de plásticos hermeticamente fechados. De cada amostra moída foram retiradas alíquotas para determinação de nitrogênio $(N)$, fósforo $(P)$, potássio $(K)$. As análises dos nutrientes foram realizadas segundo os padrões sugeridos por Carmo et al. (2000), no Laboratório de Nutrição de Plantas no Centro de Pesquisa do Semiárido (CPVSA) na UFERSA.

Amostras do substrato e da água utilizada na irrigação foram recolhidas e em seguida encaminhadas ao LASAP - UFERSA, para verificar a disponibilidade de nutrientes do solo e água. Os resultados da análise da água de irrigação encontram-se na Tabela 2. 
Tabela 2. Análise das águas utilizadas na irrigação de B. vulgaris ( $0 \%$ - água sem diluição do resíduo da piscicultura; $10 \%$ - água com diluição de $10 \%$ do resíduo da piscicultura; $15 \%$ - água com diluição de $15 \%$ do resíduo da piscicultura; $20 \%$ - água com diluição de $20 \%$ do resíduo da piscicultura; $25 \%$ - água com diluição de $25 \%$ do resíduo da piscicultura)

\begin{tabular}{llllllllllllll}
\hline Água & $\begin{array}{l}\mathrm{pH} \\
\text { (água) }\end{array}$ & $\begin{array}{l}\mathrm{CE} \\
\mathrm{dS} / \mathrm{m}\end{array}$ & $\mathrm{K}^{+}$ & $\mathrm{Na}^{+}$ & $\mathrm{Ca}^{2+}$ & $\begin{array}{c}\mathrm{Mg}^{2+} \\
(\mathrm{mmolc} / \mathrm{L})\end{array}$ & $\mathrm{Cl}^{-}$ & $\mathrm{CO}^{2-}$ & $\mathrm{HCO}_{3}^{-}$ & $\mathrm{RAS}$ & $\begin{array}{l}\text { Dureza } \\
(\mathrm{mg} / \mathrm{L})\end{array}$ & $\begin{array}{c}\text { Cátions } \\
(\mathrm{mmol} / \mathrm{L})\end{array}$ \\
\hline $0 \%$ & 8,50 & 0,61 & 0,23 & 3,34 & 0,80 & 0,50 & 2,20 & 0,60 & 2,40 & 4,1 & 65 & 4,9 & 5,2 \\
$10 \%$ & 8,40 & 4,44 & 0,61 & 28,68 & 6,00 & 6,60 & 30,00 & 0,80 & 2,80 & 11,4 & 630 & 41,9 & 33,6 \\
$15 \%$ & 8,40 & 6,18 & 0,81 & 44,99 & 7,50 & 11,30 & 42,00 & 0,80 & 2,60 & 14,7 & 940 & 64,6 & 45,4 \\
$20 \%$ & 8,50 & 7,76 & 1,04 & 43,27 & 9,10 & 16,00 & 54,00 & 1,00 & 2,60 & 12,2 & 1255 & 69,4 & 57,6 \\
$25 \%$ & 8,50 & 9,21 & 1,24 & 54,43 & 10,10 & 20,60 & 66,00 & 1,00 & 3,00 & 13,9 & 1535 & 86,4 & 70,0 \\
\hline
\end{tabular}

As análises estatísticas foram executadas com 0 auxílio dos softwares CALC e SISVAR. Os dados coletados foram submetidos a análise de variância e teste de Tukey a $5 \%$ de significância.

\section{RESULTADOS}

Aos 28 dias após o início do experimento, as variáveis fitomassa seca de folhas e raízes, nitrogênio, fósforo e sódio de $B$. vulgaris revelam interação significativa $(F<0,05)$ entre os fatores período de irrigação e utilização de água residuária da piscicultura na irrigação. Na Tabela 3 e Tabela 4, observa-se para fitomassa seca de folhas, o decréscimo da média em função do aumento no teor de água residuária na irrigação, bem como do período do turno de rega, o qual a planta foi submetida. Esse resultado evidência que plantas de $B$. vulgaris não toleram ambientes com índices de salinidade superiores a 9,21 dS m-1 (Tabela 2), já que apresenta decréscimo significativo da produção de fitomassa seca de folhas, quando comparado aos demais tratamentos (Tabela 3).

Tabela 3. Avaliação da fitomassa seca de folha (F. S.), nitrogênio, fósforo, sódio e potássio em folhas de Bambusa vulgaris vittata aos 28 dias após o início do experimento testando o período de irrigação.

\begin{tabular}{ccccccc}
\hline & $\mathrm{A}$ & \multicolumn{5}{c}{ Folha $\left(\mathrm{g} \cdot \mathrm{kg}^{-1}\right)$} \\
\cline { 2 - 6 } & $\%$ & $\mathrm{~F} . \mathrm{S}$. & $\mathrm{N}$ & $\mathrm{P}$ & $\mathrm{Na}$ & $\mathrm{K}$ \\
\hline & 0 & $12,9 \mathrm{Aa}$ & $21,8 \mathrm{Ba}$ & $0,7 \mathrm{Aa}$ & $3,0 \mathrm{Cb}$ & $0,5 \mathrm{Aa}$ \\
$\mathrm{C}$ & 10 & $12,5 \mathrm{Aa}$ & $26,2 \mathrm{Aa}$ & $0,6 \mathrm{Aa}$ & $8,2 \mathrm{Ba}$ & $0,8 \mathrm{Aa}$ \\
2 & 15 & $13,1 \mathrm{Aa}$ & $25,9 \mathrm{Aab}$ & $0,7 \mathrm{Aa}$ & $16,0 \mathrm{ABa}$ & $0,8 \mathrm{Aa}$ \\
$\mathrm{D}$ & 20 & $12,6 \mathrm{Aa}$ & $20,4 \mathrm{Bb}$ & $0,6 \mathrm{Aa}$ & $17,1 \mathrm{ABa}$ & $0,4 \mathrm{Aa}$ \\
& 25 & $10,3 \mathrm{Ba}$ & $22,7 \mathrm{Bb}$ & $0,8 \mathrm{Aa}$ & $22,7 \mathrm{Aa}$ & $0,3 \mathrm{Aa}$ \\
\hline & 0 & $13,3 \mathrm{Aa}$ & $23,0 \mathrm{Baa}$ & $0,6 \mathrm{Ab}$ & $8,9 \mathrm{Ca}$ & $0,7 \mathrm{Ba}$ \\
& 10 & $10,3 \mathrm{ABab}$ & $23,9 \mathrm{Ba}$ & $0,6 \mathrm{Aa}$ & $8,2 \mathrm{Ca}$ & $0,6 \mathrm{Ba}$ \\
7 & 15 & $8,78 \mathrm{Bb}$ & $28,2 \mathrm{Aa}$ & $0,5 \mathrm{ABb}$ & $15,7 \mathrm{Ba}$ & $0,3 \mathrm{Aa}$ \\
$\mathrm{D}$ & 15 & $8,70 \mathrm{Bb}$ & $29,4 \mathrm{Aa}$ & $0,4 \mathrm{Bb}$ & $16,9 \mathrm{Ba}$ & $0,4 \mathrm{Ba}$ \\
& 20 & $7,20 \mathrm{Bb}$ & $27,1 \mathrm{Aa}$ & $0,4 \mathrm{Bb}$ & $19,7 \mathrm{Aa}$ & $0,9 \mathrm{Bb}$ \\
\hline & 25 & $7,05 \mathrm{ABb}$ & $25,3 \mathrm{Aa}$ & $0,5 \mathrm{Ab}$ & $9,9 \mathrm{Ba}$ & $0,6 \mathrm{Aa}$ \\
& 0 & $7,55 \mathrm{ABb}$ & $29,7 \mathrm{Ba}$ & $0,5 \mathrm{Ab}$ & $8,4 \mathrm{a}$ & $0,5 \mathrm{Aa}$ \\
$\mathrm{C}$ & 10 & $10,7 \mathrm{Aa}$ & $23,9 \mathrm{Aa}$ & $0,5 \mathrm{Ab}$ & $9,7 \mathrm{~b}$ & $0,6 \mathrm{Aa}$ \\
$\mathrm{D}$ & 15 & $7,5 \mathrm{ABb}$ & $25,9 \mathrm{Aa}$ & $0,4 \mathrm{Ab}$ & $13,4 \mathrm{Ab}$ & $0,7 \mathrm{Aa}$ \\
& 20 & $6,5 \mathrm{Bb}$ & $28,0 \mathrm{Ba}$ & $0,5 \mathrm{Ab}$ & $14,2 \mathrm{Ab}$ & $0,3 \mathrm{Aa}$ \\
\hline
\end{tabular}

$\mathrm{R}=$ rega; $\mathrm{C2D}=$ cada 2 dias; $\mathrm{C7D}=$ cada 7 dias; $\mathrm{C} 14 \mathrm{D}=$ Cada 14 dias; $\mathrm{A} \%=$ porcentagem de diluição da água; F.S.=fitomassa seca; $\mathrm{N}=$ Nitrogênio= Fósforo; $\mathrm{Na}=$ Sódio; $\mathrm{K}=$ Potássio.

Médias seguidas de mesma letra maiúscula, dentro de um mesmo período de irrigação, e as seguidas de mesma letra minúscula, na mesma proporção de rejeito de água e diferentes períodos de irrigação, não diferem entre si, pelo teste de Tukey a $5 \%$ de probabilidade. 
O estresse hídrico também proporciona redução na fitomassa seca das folhas. Porém, o efeito conjunto do estresse hídrico e salino proporciona decréscimo ainda maior na produção de fitomassa seca na parte foliar, o que indica que a espécie não tolera períodos maiores que 7 dias com deficiência hídrica e, se, a água utilizada na irrigação for salina, a perda de fitomassa é significativamente superior (Tabela 3). Contudo, quando se observa a fitomassa seca de raízes, tem- se maior média para essa variável nos tratamentos que são irrigados a cada dois dias com maior teor de água residuária, com consequente aumento no teor da salinidade. Esse aumento na fitomassa seca de raiz, quando a planta é submetida à salinidade, pode indicar que a espécie estudada pode ser tolerante ao estresse salino. Todavia, quando as plantas são submetidas ao estresse hídrico e salino concomitantemente, observase decréscimo na fitomassa seca das raízes (Tabela 4).

Tabela 4. Fitomassa seca de raiz (F. S.), nitrogênio, fósforo, sódio e potássio em raízes de Bambusa vulgaris vittata aos 28 dias após o início do experimento testando o período de irrigação.

\begin{tabular}{|c|c|c|c|c|c|c|}
\hline \multirow{2}{*}{$\mathrm{R}$} & \multirow{2}{*}{$\begin{array}{l}\text { A } \\
\%\end{array}$} & \multicolumn{5}{|c|}{ Raiz $\left(\mathrm{g} \cdot \mathrm{kg}^{-1}\right)$} \\
\hline & & F.S. & $\mathrm{N}$ & $\mathrm{P}$ & $\mathrm{Na}$ & K \\
\hline \multirow{5}{*}{$\begin{array}{l}C \\
2 \\
D\end{array}$} & 0 & $9,4 \mathrm{Bb}$ & $18,6 \mathrm{Ba}$ & $0,8 \mathrm{Aa}$ & $2,7 \mathrm{Ba}$ & $0,3 \mathrm{Aa}$ \\
\hline & 10 & $12,1 \mathrm{ABa}$ & $21,0 \mathrm{ABa}$ & $0,5 \mathrm{ABb}$ & $4,0 \mathrm{ABa}$ & $0,5 \mathrm{Aa}$ \\
\hline & 15 & $12,3 \mathrm{ABa}$ & $17,7 \mathrm{Bb}$ & $0,3 \mathrm{ABa}$ & $6,4 \mathrm{ABa}$ & $0,2 \mathrm{Aa}$ \\
\hline & 20 & $12,1 \mathrm{ABa}$ & $20,1 \mathrm{ABa}$ & $0,1 \mathrm{Bb}$ & $6,8 \mathrm{ABa}$ & $0,9 \mathrm{Bb}$ \\
\hline & 25 & $13,4 \mathrm{Aa}$ & $21,8 \mathrm{ABa}$ & $0,1 \mathrm{Bb}$ & $7,5 \mathrm{Aa}$ & $0,5 \mathrm{Aa}$ \\
\hline \multirow{5}{*}{$\begin{array}{l}C \\
7 \\
D\end{array}$} & 0 & $10,4 \mathrm{ABb}$ & $19,2 \mathrm{BCa}$ & $0,4 \mathrm{ABb}$ & $2,9 \mathrm{Ba}$ & $0,6 \mathrm{Bb}$ \\
\hline & 10 & $6,1 \mathrm{Bb}$ & $26,2 \mathrm{ABa}$ & $0,7 \mathrm{Aa}$ & $2,8 \mathrm{Aab}$ & $0,4 \mathrm{ABb}$ \\
\hline & 15 & $8,7 \mathrm{ABb}$ & $30,6 \mathrm{Aa}$ & $0,2 \mathrm{Bb}$ & $5,5 \mathrm{ABa}$ & $0,2 \mathrm{Aa}$ \\
\hline & 20 & $10,4 A B$ & $18,4 \mathrm{Ca}$ & $0,2 \mathrm{Bab}$ & $5,6 \mathrm{ABa}$ & $0,1 \mathrm{Aa}$ \\
\hline & 25 & $12,9 \mathrm{Aa}$ & $27,7 \mathrm{ABa}$ & $0,3 \mathrm{ABa}$ & $6,7 \mathrm{Aa}$ & $0,4 \mathrm{ABb}$ \\
\hline \multirow{5}{*}{$\begin{array}{c}C \\
14 \\
D\end{array}$} & 0 & $12,1 \mathrm{Aa}$ & $21,3 \mathrm{Aa}$ & $0,6 \mathrm{Aa}$ & $2,4 \mathrm{Ba}$ & $0,1 \mathrm{Aa}$ \\
\hline & 10 & 9,0ABab & $19,2 \mathrm{Aa}$ & $0,4 \mathrm{Ab}$ & $3,5 \mathrm{Ba}$ & $0,2 \mathrm{Aa}$ \\
\hline & 15 & $8,6 \mathrm{ABb}$ & $16,04 \mathrm{ABb}$ & $0,4 \mathrm{Aa}$ & $5,0 \mathrm{Aa}$ & $0,4 \mathrm{Aa}$ \\
\hline & 20 & $8,2 \mathrm{ABb}$ & $20,71 \mathrm{Aa}$ & $0,6 \mathrm{Aa}$ & $5,2 \mathrm{Aa}$ & $0,3 \mathrm{Aa}$ \\
\hline & 25 & $10,9 \mathrm{ABb}$ & $10,21 \mathrm{Bb}$ & $0,2 \mathrm{Bab}$ & $5,8 \mathrm{Aa}$ & $0,1 \mathrm{Aa}$ \\
\hline
\end{tabular}

$\mathrm{R}=$ rega; $\mathrm{C} 2 \mathrm{D}=$ cada 2 dias; $\mathrm{C7D}=$ cada 7 dias; $\mathrm{C} 14 \mathrm{D}=$ Cada 14 dias; $\mathrm{A} \%=$ porcentagem de diluição da água; F.S.=fitomassa seca; $\mathrm{N}=$ Nitrogênio = Fósforo; $\mathrm{Na}=$ Sódio; $\mathrm{K}=$ Potássio .

Médias seguidas de mesma letra maiúscula, dentro de um mesmo período de irrigação, e as seguidas de uma mesma letra minúscula na mesma proporção de rejeito de água e diferentes períodos de irrigação não diferem entre si, pelo teste de Tukey a $5 \%$ de probabilidade.

O teor de nitrogênio e fósforo, tanto na parte aérea quanto nas raízes, apresentam níveis médios e comportamento aleatório, conforme a interação entre períodos de irrigação e uso de água da piscicultura na irrigação. Esses resultados demonstram que esses nutrientes estavam sendo absorvidos pelas plantas e estavam sendo transcolados para a parte aérea (Tabela 4). Por outro lado, observa-se que o nível de sódio presente nas raízes e parte área apresenta incremento significativo com o aumento na proporção da água residuária aplicada na irrigação das plantas. Todavia, há redução no teor de sódio quando as irrigações são atenuadas, a exemplo do observado no teor de sódio nas raízes e folhas de plantas irrigadas a cada 14 dias (Tabela 4). Esse resultado ocorre em função dos altos níveis de sódio presente na água residuária, conforme apresentado na Tabela 2.

Aos 28 dias, após a condução do experimento, foi realizada a análise de solo dos tratamentos testados. É possível observar que o teor de Nitrogênio total no solo teve um leve incremento à medida que aumenta a proporção de água residuária na irrigação. Porém, quando reduz as irrigações, a exemplo da realizada a cada 14 dias, não há incremento no teor nutricional em relação ao tratamento que não faz uso da água residuária. A maior proporção de nitrogênio nos tratamentos com maior proporção de água residuária está relacionado com a concentração desse nutriente na água utilizada. Por outro lado, o pH do solo mantémse com pouca alteração independente da utilização da água residuária ou do período em que foram realizadas 
as irrigações. Contudo, a condutividade elétrica do solo apresentou incremento significativo quando o teor da água residuária utilizada na irrigação das plantas aumentou, conforme a Tabela 5.

Tabela 5. Análise de solo aos 28 dias após o início do experimento testando o período de írrigação (a cada dois, sete e 14 dias) e a utilização de água residuária com diluição de $(0,10,15,20$ e $25 \%$ ) - na irrigação de mudas de B. vulgaris.

\begin{tabular}{|c|c|c|c|c|c|c|c|c|c|c|c|c|c|c|}
\hline \multirow{2}{*}{$\mathbf{R}$} & \multirow{2}{*}{$\begin{array}{l}A ́ \\
\%\end{array}$} & \multirow{2}{*}{$\begin{array}{c}N \\
g / k g\end{array}$} & \multirow{2}{*}{$\mathrm{pH}$} & \multirow{2}{*}{$\begin{array}{c}C E \\
\mathrm{dS} . \mathrm{m}_{-1}\end{array}$} & \multirow{2}{*}{$\begin{array}{l}\text { Mat,Or } \\
\text { g.kg-1 }\end{array}$} & P & $\mathrm{K}^{+}$ & $\mathrm{Na}^{+}$ & $\mathrm{Ca}^{2+}$ & SB & $t$ & CTC & V & PST \\
\hline & & & & & & & $\mathrm{mg} \cdot \mathrm{dm}^{-3}$ & & \multicolumn{4}{|c|}{ cmolc. $\mathrm{dm}^{-3}$} & \multicolumn{2}{|c|}{$\%$} \\
\hline \multirow{5}{*}{$\begin{array}{l}\text { C } \\
2 \\
D\end{array}$} & 0 & 0,53 & 8,6 & 0,28 & 11,3 & 93,6 & 101,8 & 134,4 & 4,2 & 378 & 378 & 378 & 100 & 35,5 \\
\hline & 10 & 0,67 & 8,0 & 0,62 & 11,8 & 115,8 & 125,2 & 498,3 & 4,5 & 742 & 742 & 742 & 100 & 67,2 \\
\hline & 15 & 0,98 & 8,2 & 0,75 & 14,7 & 147,1 & 131,2 & 758,6 & 4,7 & 1093 & 1093 & 1093 & 100 & 69,4 \\
\hline & 20 & 1,05 & 8,3 & 1,50 & 14,3 & 88,4 & 132,2 & 788,6 & 4,4 & 1039 & 1039 & 1039 & 100 & 75,9 \\
\hline & 25 & 0,91 & 8,0 & 1,92 & 11,7 & 129,1 & 158,0 & 998,8 & 4,2 & 1286 & 1286 & 1286 & 100 & 77,7 \\
\hline \multirow{5}{*}{$\begin{array}{l}\text { C } \\
7 \\
\text { D }\end{array}$} & 0 & 0,74 & 8,6 & 0,30 & 13,2 & 114,3 & 114,3 & 134,4 & 4,5 & 401 & 401 & 401 & 100 & 33,6 \\
\hline & 10 & 1,02 & 8,1 & 0,42 & 12,6 & 101,2 & 122,7 & 373,2 & 5,5 & 657 & 657 & 657 & 100 & 56,8 \\
\hline & 15 & 0,67 & 7,9 & 0,72 & 11,8 & 97,8 & 136,2 & 503,3 & 4,7 & 764 & 764 & 764 & 100 & 65,9 \\
\hline & 20 & 1,02 & 7,8 & 1,20 & 8,3 & 78,9 & 139,1 & 558,3 & 5,1 & 800 & 800 & 800 & 100 & 69,8 \\
\hline & 25 & 2,42 & 8,0 & 1,24 & 13,0 & 107,2 & 154,5 & 663,5 & 4,2 & 911 & 911 & 911 & 100 & 72,8 \\
\hline \multirow{5}{*}{$\begin{array}{c}C \\
14 \\
D\end{array}$} & 0 & 0,81 & 8,1 & 0,26 & 13,6 & 98,5 & 117,3 & 156,9 & 5,6 & 401 & 401 & 401 & 100 & 39,1 \\
\hline & 10 & 0,81 & 8,0 & 0,37 & 20,7 & 109,4 & 163,5 & 278,1 & 4,9 & 606 & 606 & 606 & 100 & 45,9 \\
\hline & 15 & 0,88 & 8,1 & 0,52 & 14,9 & 115,1 & 178,9 & 318,1 & 5,9 & 744 & 744 & 744 & 100 & 54,9 \\
\hline & 20 & 0,91 & 8,1 & 0,77 & 10,4 & 124,1 & 183,9 & 408,2 & 5,2 & 631 & 631 & 631 & 100 & 50,4 \\
\hline & 25 & 0,88 & 8,0 & 0,90 & 9,4 & 97,39 & 189,8 & 458,3 & 6,8 & 760 & 760 & 760 & 100 & 60,3 \\
\hline
\end{tabular}

$R=$ rega; $C 2 D=$ cada 2 dias; $C 7 D=$ cada 7 dias; $C 14 D=c a d a ~ 14$ dias; $A \%=$ porcentagem de diluição da água;

As variáveis, condutividade elétrica, íons potássio e íons de sódio no solo aos 28 dias após 0 início do experimento, revelam interação significativa $(F<0,05)$ entre os fatores período de irrigação e utilização de água residuária da piscicultura na irrigação (Tabelas 3 e 4). A condutividade elétrica é superior nos tratamentos em que se utiliza a água residuária em maior proporção. 0 que já era esperado, pois a análise das águas utilizadas na irrigação revelou que o tratamento com maior proporção de água advinda da piscicultura tem maior condutividade elétrica (Tabela 2).

Assim, à medida que se realizava as irrigações, parte dos sais eram acumulados no solo e outros íons eram absorvidos pelas plantas (Tabelas 3 e 4). Por outro lado, à medida que se reduz as irrigações, reduz também a condutividade elétrica do solo (Tabela 5) já que não há deposição em grande quantidade de sais ao solo.

Para o potássio, também se observa um incremento no teor do nutriente com o aumento na proporção da água residuária na irrigação. Médias superiores são observadas quando as plantas são submetidas ao estresse hídrico por 14 dias. Todavia, os íons de sódio presente no solo são significativamente superiores na maior proporção de água residuária e com a irrigação sendo realizados a cada dois dias. Esse resultado é observado em função da maior concentração de íons de sódio na água residuária como também na maior quantidade de água aplicada quando comparado com os outros tratamentos (Tabela 3).

Para o íon cálcio presente no solo, foi observado um aumento em seus níveis a partir da análise inicial do solo (Tabela 1). Com o aumento, percebe-se que parte foi acumulada no solo e outra parte foi utilizada pela planta, ficando estabilizado em uma faixa de 4 a 5 cmolc.dm3 em todos os tratamentos. Nas plantas que foram submetidas à irrigação com a dose de $25 \%$ aos 14 dias, observou-se um significativo aumento. Grande parte de produção de fitomassa de raízes observada nos tratamentos com elevada salinidade (Tabela 4) pode ter sido influenciada pela absorção de cálcio, não ficando grandes proporções retidas no solo, pois podemos observar na Tabela 2, índices consideráveis de cálcio na água de irrigação. 
A relação $\mathrm{Na}+/ \mathrm{K}+$ (Tabela 6) expressa incremento em relação ao aumento nos níveis de sais na água de irrigação. Os valores foram bem superiores nas raízes do que nas folhas, e isso se deve mais ao elevado acúmulo de sódio do que a redução na absorção de potássio. Porém, os valores da relação $\mathrm{N}+/ \mathrm{K}+$ não foram superiores a $1 \mathrm{~g} \cdot \mathrm{kg}^{-1}$, indicando que essa variável não foi determinante na redução do desenvolvimento da planta e na taxa de fotossíntese.

Tabela 6. Condutividade elétrica (CE), potássio e íons de sódio no solo aos 28 dias após o início do experimento testando o período de irrigação (a cada dois, sete e 14 dias) e a utilização de água residuária com diluição de 0,10 , 15,20 e $25 \%$,- na irrigação de mudas de B. vulgaris.

\begin{tabular}{|c|c|c|c|c|}
\hline$R$ & $\begin{array}{c}A \\
(\%)\end{array}$ & $\begin{array}{c}C E \\
d S \cdot m^{-1}\end{array}$ & $\begin{array}{c}\mathrm{K}^{+} \\
\mathrm{mg} \cdot \mathrm{dm}^{-3}\end{array}$ & $\begin{array}{c}\mathrm{Na}^{+} \\
\mathrm{mg} \cdot \mathrm{dm}^{-3}\end{array}$ \\
\hline & 0 & $0,28 \mathrm{Aa}$ & $101,86 \mathrm{Aa}$ & $134,42 \mathrm{Aa}$ \\
\hline C & 10 & $0,62 \mathrm{Ba}$ & $125,23 \mathrm{Ba}$ & 498,33 Ba \\
\hline 2 & 15 & $0,75 \mathrm{Ba}$ & $131,19 \mathrm{BCa}$ & $758,60 \mathrm{Ca}$ \\
\hline \multirow[t]{3}{*}{ D } & 20 & $1,50 \mathrm{Ca}$ & $132,18 \mathrm{BCa}$ & $788,63 \mathrm{Ca}$ \\
\hline & 25 & $1,92 \mathrm{Ca}$ & $158,03 \mathrm{Ca}$ & $998,84 \mathrm{Da}$ \\
\hline & 0 & $0,30 \mathrm{Aa}$ & $114,29 \mathrm{Aa}$ & $134,42 \mathrm{Aa}$ \\
\hline C & 10 & $0,42 \mathrm{Ab}$ & $122,74 \mathrm{ABa}$ & $373,20 \mathrm{Ba}$ \\
\hline 7 & 15 & $0,72 \mathrm{ABa}$ & $136,16 \mathrm{ABa}$ & $503,34 \mathrm{Cb}$ \\
\hline \multirow[t]{3}{*}{ D } & 20 & $1,20 \mathrm{Bb}$ & $139,14 \mathrm{ABa}$ & $558,39 \mathrm{Cb}$ \\
\hline & 25 & $1,24 \mathrm{Bb}$ & $154,55 \mathrm{Ba}$ & $663,50 \mathrm{Db}$ \\
\hline & 0 & $0,26 \mathrm{Aa}$ & $117,27 \mathrm{Aa}$ & $116,94 \mathrm{Aa}$ \\
\hline$C$ & 10 & $0,37 A b$ & $163,50 \mathrm{Bb}$ & $278,11 \mathrm{Bb}$ \\
\hline 14 & 15 & $0,52 \mathrm{ABb}$ & $178,91 \mathrm{Bb}$ & $318,15 \mathrm{BC}$ \\
\hline \multirow[t]{2}{*}{$D$} & 20 & $0,77 A b c$ & $183,88 \mathrm{Bb}$ & $408,24 \mathrm{CDC}$ \\
\hline & 25 & $0,90 \mathrm{Bc}$ & $189,85 \mathrm{Bb}$ & $458,29 D \mathrm{c}$ \\
\hline
\end{tabular}

$\mathrm{R}=$ rega; $\mathrm{C} 2 \mathrm{D}=$ cada 2 dias; $\mathrm{C} 7 \mathrm{D}=$ cada 7 dias; $\mathrm{C} 14 \mathrm{D}=$ cada 14 dias; $\mathrm{A} \%=$ =porcentagem de diluição da água;

Médias seguidas de mesma letra maiúscula, dentro de um mesmo período de irrigação, e as seguidas de mesma letra minúscula na mesma proporção de rejeito de água residuária da piscicultura e diferentes períodos de irrigação, não diferem entre si, pelo teste de Tukey a $5 \%$ de probabilidade.

Para os teores de clorofila a, observa-se que houve aumento tanto para as plantas irrigadas com a concentração de $20 \%$ de água residuária a cada 7 dias quanto 14 dias. Para o teor de clorofila b, os menores valores foram observados no ciclo de rega a cada 7 dias e em relação ao aumento da condutividade elétrica, estes valores foram afetados apenas para os ciclos de rega a cada 2 dias, a partir de $20 \%$ de água residuária, e 7 dias a partir de 15\%, sendo estabilizado aos 14 dias onde todos tratamentos foram semelhantes. Foi observada para os carotenoides uma maior sensibilidade em função ao estresse hídrico do que ao aumento da salinidade, uma vez que houve uma redução de 31\% para as plantas irrigadas a cada 14 dias para as plantas irrigadas com $0 \%$ de água residuária (Tabela 7 ).

\section{DISCUSSÃO}

A tolerância à salinidade tem sido correlacionada com a manutenção de uma adequada nutrição potássica. Logo, a relação $\mathrm{Na}+/ \mathrm{K}+$ tem sido considerada como um parâmetro ou critério de seleção de genótipos mais resistentes à salinidade (Alves et al. 2008).

Os declínios nos teores de clorofila são resultados dos desequilíbrios nas atividades fisiológicas e bioquímicas promovidas pelo teor de sais, além do tolerado pelas culturas. 0 excesso de sais estimula a atividade enzimática da clorofilase que degrada as moléculas do pigmento fotossintetizante e induz a destruição estrutural dos cloroplastos, provocando também o desbalanceamento e perda de atividade das proteínas de pigmentação.

A utilização de água proveniente de tanque de piscicultura é interessante, pois há grande disponibilidade de fósforo e nitrogênio nesse resíduo. Com o controle adequado essa água pode ser utilizada direto na irrigação e adubação de plantas via hidroponia ou até irrigação continua observando cuidadosamente os níveis de sais para que não cause um dano solo. Vários pesquisadores estudam a viabilidade de utilização deste resíduo em culturas de tomate cereja e alface, em gramíneas também vem sendo feito estudos 
para indicar a viabilidade e 0 aumento da produção em áreas que frequentemente é manejada pelo pequeno produtor. Entender o comportamento da planta diante dessa condição salina apresentada é fundamental para determinar sua viabilidade.

O resultado encontrado por Branco (2016), em folhas de plantas de Bambusa vulgaris submetidas a níveis crescentes de sais da água de irrigação, é semelhante aos encontrados na Tabela 3. Os resultados encontrados por ele mostram que a salinidade influenciou significativamente no teor de $\mathrm{Na}+$ e a relação $\mathrm{Na} / \mathrm{K}$. Todavia, na folha, o único teor que não foi influenciado pela ação dos sais na água foi o Cl- contrariando o que foi observado nesse experimento. Os maiores teores do sódio na fitomassa seca foram encontrados nas plantas que foram expostas aos maiores teores de salinidade da água de irrigação, resultado observado também nesse trabalho. Todavia, os níveis do sódio na folha foram menores que em relação aos das raízes, verificando assim um mecanismo de retenção desse íon nos caules das plantas de bambu, evitando assim seu acúmulo nos tecidos foliares.

Ao observar seu experimento com cana de açúcar, Santos et al. (2013) verificaram que as mudas submetidas ao estresse hídrico tiveram uma redução no tamanho das folhas e do colmo da cana de açúcar, entretanto, as mudas testadas continuaram emitindo folhas novas, as quais se permaneciam turgidas. Já no $B$. vulgaris testado, o sintoma de estresse hídrico já era evidenciado aos 7 dias, onde as folhas caíram e foram emitidas folhas novas. Aos 14 dias algumas plantas entraram em murcha permanente e outras se restabeleceram após a irrigação. Esses resultados evidenciam que tanto a cana quanto 0 bambu são tolerantes ao estresse hídrico por um curto período, se mantendo vivas e diminuindo sua produção de folhas e de colmos.

OsresultadosapresentadosnaTabela4evidenciam que as plantas de $B$. vulgaris, quando submetidas somente ao estresse salino, investem na produção de raízes e conseguem manter a produção de fitomassa seca da parte área com até $20 \%$ de água residuária na irrigação. Esses resultados também evidenciam que o estresse hídrico por período acima de sete dias pode induzir a planta a perda de fitomassa radicular e foliar.

As reduções nos teores de clorofila, observadas na Tabela 7, segundo Neves e Spat (2013), deve-se ao fato da existência de competição da molécula de sódio, em dissolução, com a de magnésio na formação da clorofila. Tawfik (2008) constatou redução progressiva dos pigmentos fotossintéticos (clorofila a, btotal e carotenoides) no feijão Vigna quando submetidos a estresse salino de 50 e $75 \mathrm{mM}$ de $\mathrm{NaCl}$. Porém, em contraste com o que foi observado por esses pesquisadores, a redução de clorofila neste trabalho foi mais expressiva em função do estresse hídrico, não em função da salinidade.

Tabela 7. Clorofila a, Clorofila b e carotenoides em folhas de mudas de $B$. vulgaris, aos 28 dias, após o início do experimento, testando o período de irrigação (a cada dois, sete e 14 dias) e a utilização de água residuária com diluição de 0,10, 15, 20 e $25 \%$.

\begin{tabular}{|c|c|c|c|c|}
\hline $\mathrm{R}$ & $\begin{array}{c}A \\
(\%)\end{array}$ & $\begin{array}{c}\text { Clorofila a } \\
\left(\mathrm{mg} \cdot \mathrm{g}^{-1}\right)\end{array}$ & $\begin{array}{c}\text { Clorofila b } \\
\left(\mathrm{mg} \cdot \mathrm{g}^{-1}\right)\end{array}$ & $\begin{array}{c}\text { Carotenoides } \\
\left(\mathrm{mg} \cdot \mathrm{g}^{-1}\right)\end{array}$ \\
\hline \multirow{5}{*}{ C 2 D } & 0 & $0,42 \mathrm{Ab}$ & $0,42 \mathrm{Aa}$ & 0,26 Aab \\
\hline & 10 & $0,49 \mathrm{Aa}$ & $0,44 \mathrm{Aab}$ & $0,35 \mathrm{Aa}$ \\
\hline & 15 & $0,36 \mathrm{Aa}$ & $0,32 \mathrm{Aa}$ & $0,26 \mathrm{Aa}$ \\
\hline & 20 & $0,41 \mathrm{Ab}$ & $0,19 \mathrm{Bb}$ & $0,27 \mathrm{Aa}$ \\
\hline & 25 & $0,51 \mathrm{Aa}$ & $0,23 \mathrm{Ba}$ & $0,26 \mathrm{Aa}$ \\
\hline \multirow{5}{*}{ C 7 D } & 0 & $0,39 \mathrm{Ba}$ & $0,37 \mathrm{Ba}$ & $0,38 \mathrm{Aa}$ \\
\hline & 10 & $0,67 \mathrm{Bb}$ & $0,67 \mathrm{Aa}$ & $0,30 \mathrm{Aa}$ \\
\hline & 15 & 0,34 Ba & $0,36 \mathrm{Ba}$ & $0,17 \mathrm{Bb}$ \\
\hline & 20 & $0,48 \mathrm{Aa}$ & $0,20 \mathrm{Bb}$ & $0,26 \mathrm{Aa}$ \\
\hline & 25 & $0,59 \mathrm{Ba}$ & $0,23 \mathrm{Ba}$ & $0,29 \mathrm{Aa}$ \\
\hline \multirow{5}{*}{ C $14 \mathrm{D}$} & 0 & $0,61 \mathrm{Ba}$ & $0,57 \mathrm{Aa}$ & $0,18 \mathrm{Bb}$ \\
\hline & 10 & $0,38 \mathrm{Bb}$ & $0,33 \mathrm{Ab}$ & $0,30 \mathrm{Aa}$ \\
\hline & 15 & $0,56 \mathrm{Ba}$ & $0,51 \mathrm{Aa}$ & $0,30 \mathrm{Aa}$ \\
\hline & 20 & $1,03 \mathrm{Aa}$ & $0,43 \mathrm{Aa}$ & $0,31 \mathrm{Aa}$ \\
\hline & 25 & $0,57 \mathrm{Ba}$ & $0,33 \mathrm{Aa}$ & $0,33 \mathrm{Aa}$ \\
\hline
\end{tabular}

$R=$ rega; $C 2 D=$ cada 2 dias; $C 7 D=$ cada 7 dias; $C 14 D=c a d a ~ 14$ dias; $A \%=$ porcentagem de diluição da água; Médias seguidas de mesma letra maiúscula, dentro de um mesmo período de irrigação, e as seguidas de mesma letra minúscula na mesma proporção de rejeito de água residuária da piscicultura e diferentes períodos de irrigação não diferem entre si, pelo teste de Tukey a 5\% de probabilidade. 
As quantificações de conteúdo de clorofilas em folhas de plantas são utilizadas em estudos fisiológicos para avaliar o crescimento e o desenvolvimento das plantas. Estas variáveis são importantes no estudo do comportamento vegetativo de espécies vegetais e na resposta das plantas às técnicas de manejo que visam a aumentar o potencial fotossintético, principalmente relacionadas aos sistemas de condução e adaptabilidade às condições do ambiente (Amarante et al., 2009).

$\mathrm{Na}$ água de irrigação proveniente da piscicultura, os níveis de nutrientes essências para a planta são importantes para se entender a tolerância dela a regas consecutivas. Os níveis elevados de fósforo e potássio contribuíram significativamente para manutenção das raízes até a dose de $20 \%$ de água residuária, mostrando em condições que se mantenha a irrigação, que a salinidade da água testada foi insignificante para $\circ$ B. vulgaris vittata em relação a sua sobrevivência e o desenvolvimento nutricional é beneficiado pela irrigação continua com essa água, dentro da dosagem que não é letal.

As pesquisas em torno do tema de reaproveitamento de água de piscicultura tentam ver a possibilidade de irrigação e adubação das culturas, observando o incremento de massa e resistência das mesmas, $0 B$. vulgaris pode ser uma alternativa ao pequeno produtor que pretende fazer um manejo da espécie em regiões com restrição hídrica, podendo assim manter o cultivo permanente. Desta forma, com a avaliação desses resultados, podemos considerar que plantas de $B$. vulgaris reduz a produção de fitomassa foliar e mantém a produção de fitomassa da raiz e os teores de pigmentos fotossintéticos, sendo mais sensível ao estresse hídrico do que ao estresse salino.

0 estresse hídrico afetou a absorção dos macronutrientes independente da concentração de água residuária avaliada. As mudas de $B$. vulgaris podem ser irrigadas com água residuária até a concentração de $25 \%$, correspondendo a uma condutividade elétrica de 9 dS.m-1, bem como com regas intercaladas a cada sete dias.

\section{REFERÊNCIAS BIBLIOGRÁFICA}

Almeida, J. P. N. de; Freitas, M. O. de; Nogueira, N. W; Oliveira, F. de A. de; Ferreira, H.; Leite, M. de S. Production of Piptadenia stipulacea (Benth.) Ducke seedlings irrigated with fish farming wastewater. Revista Brasileira de Engenharia Agrícola e Ambiental, 2017, 21, 6, 386-391.

Almeida, R. A.; Silva Junior, E. D. Tratamento de águas residuária em solo vegetado com bambu. In: Patrícia Maria Drummond; Guilherme Wiedman. (Org.). Bambus no Brasil: da biologia à tecnologia. Instituto Ciência Hoje, 2017, 641-655.

Alves F. A. L; Silva, S. L. F. da; Lima, J. P. M. S.; Silveira, J. A. G. da. Efeitos do $\mathrm{KCl}$ e $\mathrm{CaCl} 2$ na absorção e transporte de $\mathrm{Na}+$ em cajueiro exposto a salinidade. Revista Ciência Agronômica, 2008, 2, 39, 287-294.

Amarante, C. V. T. do; Zanardi, O. Z; Miqueloto, A.; Steffens, C. A.; Erhart, J.; Almeida, J. A. de. Quantificação da área e do teor de clorofilas em folhas de plantas jovens de videira Cabernet Sauvi. Revista Brasileira de Fruticultura, 2009, 31, 3, 680-686.

Assis Júnior, J. O. de; Lacerda, C. F. de; Silva, F. B. da; Silva, F. B. da; Bezerra, M. A; Gheyi, H. R. Produtividade do feijão-de-corda e acúmulo de sais no solo em função da fração de lixiviação e da salinidade da água de irrigação. Engenharia Agrícola, 2007, 27, 3, 702-713.

Baioni, J. C.; Squassoni, G.H.; Cultri, G. R. de S.; Silva, J. D.T. da; Dias, L. T. S. Fish farming effluents in production of chive-coriander consortium. Nucleus Animalium, Ituverava, v. 9, n. 1, p.143-150, 30 nov. 2017. Fundacao Educational de Ituverava. Branco, L. M. C. Produção de mudas a partir de rizomas de $B$. vulgaris sob irrigação com água salina. 2016. 79f. Tese Doutorado - Universidade Federal do Ceará, Fortaleza. 2016.

Brasil. Lei $n^{0}$ 12.484, de 8 de setembro de 2011. Dispõe sobre a Política Nacional de Incentivo ao Manejo Sustentado e ao Cultivo do Bambu e dá outras providências. Diário Oficial [da] República Federativa do Brasil, Brasília, DF, 9 set. 2011. Disponível em: <http:// www.planalto.gov.br/ccivil_03/_ato2011-2014/2011/lei/ |12484.htm>. Acesso em: 11 out. 2018.

Carmo, C. A. F. de S; Araújo, W. S. de; Bernardi, A. C. de C; Saldanha, M. F. C. Métodos de análise de tecidos vegetais utilizados na Embrapa Solos. Embrapa Solos. Circular Técnica, 2000, nº 6, $41 \mathrm{p}$. 
Embrapa. Empresa brasileira de pesquisa agropecuária -. Centro Nacional de Pesquisa de solos. Sistema brasileiro de classificação de solos. 2. ed. Embrapa, 2006. 306p.

Filgueiras, T. S. Viana, P. L. Bambus nativos: morfologia, taxonomia, distribuição e conservação. In: Patrícia M. Drummond; Guilherme Wiedman. (Org.). Bambus no Brasil: da biologia à biotecnologia. 1ed. Rio de Janeiro: Instituto Ciência Hoje, 2017, 1, 10-27.

Guimarães, I. P; Oliveira, F. de A. de; Torres, S. B.; Pereira, F. EBC.; França, F.D. de; Oliveira, M. KT. Use of fish-farming wastewater in lettuce cultivation. Revista Brasileira de Engenharia Agrícola e Ambiental, 2016, 20, 8, 728-733.

Lichtenthaler, H. K.; buschmann, C. Chlorophylls and Carotenoids: Measurement and Characterization by UV-VIS Spectroscopy. Current Protocols In Food Analytical Chemistry, 2001, 1, 1, 431-438.

Marengo, J. A.; Cunha, A. P.; Alves, L. M. A seca de 2012-15 no semiárido do Nordeste do Brasil no contexto histórico. 2016. Disponível em: http://climanalise.cptec. inpe.br/ rclimanl/revista/pdf/30anos/marengoetal.pdf. Acesso em: 11 out. 2018.

Neves, L. A. S. das; Spat, C. Concentração de clorofila e de prolina em genótipos de arroz submetidos à salinidade. Revista Unimontes Científica, 2013, 15, 1, 2013.

Pereira, M. A. R.; beraldo, A. L. Bambu de corpo e alma. 2. ed. Bauru: Canal 6, 2008. 240 p.
Santana, G. M.; Lelis, R. C. C.; Jaguaribe, E. F.; Morais, R de. M.; Paes, J. B.; Trugilho, P. F. Development of activated carbon from bamboo ( $B$. vulgaris) for pesticide removal from aqueous solutions. Cerne, 2017, 23, 1, 123-132.

Santos, W de. O.; Mesquita, F de. O.; Batista, B. D de. O.; Batista, R. O.; Alves, A de. S. Precipitações máximas para o município de Mossoró de 1964 a 2011 pela distribuição de gumbel. Brazilian Journal of Irrigation and Drainage - IRRIGA, 2014, 19, 2, 207-213.

Santos, H. R. B.; Pedrosa, E M. R.; Nogueira, R J. M. C.; Rolim, M. M.; Maranhão, S R. V. L.; Medeiros, D. B. Crescimento de três variedades de cana-de-açúcar submetidas a estresse hídrico associado à Meloidogy. Revista Brasileira de Ciências Agrárias - Brazilian Journal Of Agricultural Sciences, 2013, 8, 4, 547-554.

Silva, José L. de A.; Medeiros, J. F. de; Alves, S. S. V.; Oliveira, F. de A. de; Junior, M. J. da S; Nascimento, I. B. do. Uso de águas salinas como alternativa na irrigação e produção de forragem no semiárido nordestino. Revista Brasileira de Engenharia Agrícola e Ambiental, 2014, 18, 10, 66-72.

Tawfik, K. M. Evaluating the use of Rhizobacterin on cowpea plants grown under salt stress. Research Journal of Agriculture and Biological Sciences, 2018, 4, 26-36. Disponível em: http://www.aensiweb.net/ AENSIWEB/rjabs/rjabs/2008/26-33.pdf. 26 Acesso em: dez. 2018.

Vieira, G. G. Desertificação e convivência com o semiárido brasileiro: da casa do mocó à agroecologia e permacultura na região de Gilbués, Piauí. OKARA: Geografia em Debate, 2009, 3, 1, 23-53. 